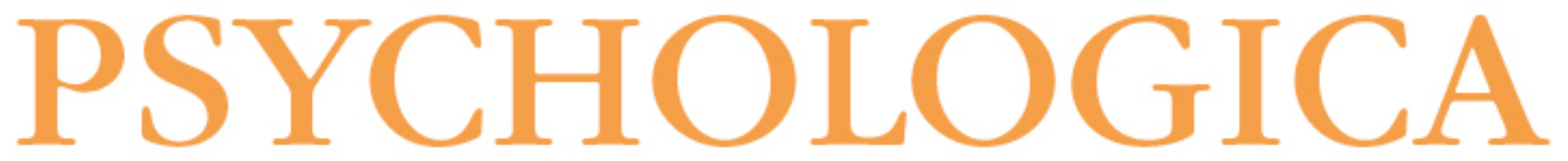

Paixão pelo trabalho e competências de gestão interpessoal: Validação de escalas para a sua avaliação junto de gestores empresariais

Autor(es): $\quad$ Martins, Margarida; Araújo, Alexandra M.; Almeida, Leandro S.

Publicado por: Imprensa da Universidade de Coimbra

URL

persistente: URI:http://hdl.handle.net/10316.2/35822

DOI: $\quad$ DOI:http://dx.doi.org/10.14195/1647-8606_57_1_4

Accessed : $\quad$ 26-Apr-2023 00:42:11

A navegação consulta e descarregamento dos títulos inseridos nas Bibliotecas Digitais UC Digitalis, UC Pombalina e UC Impactum, pressupõem a aceitação plena e sem reservas dos Termos e Condições de Uso destas Bibliotecas Digitais, disponíveis em https://digitalis.uc.pt/pt-pt/termos.

Conforme exposto nos referidos Termos e Condições de Uso, o descarregamento de títulos de acesso restrito requer uma licença válida de autorização devendo o utilizador aceder ao(s) documento(s) a partir de um endereço de IP da instituição detentora da supramencionada licença.

Ao utilizador é apenas permitido o descarregamento para uso pessoal, pelo que o emprego do(s) título(s) descarregado(s) para outro fim, designadamente comercial, carece de autorização do respetivo autor ou editor da obra.

Na medida em que todas as obras da UC Digitalis se encontram protegidas pelo Código do Direito de Autor e Direitos Conexos e demais legislação aplicável, toda a cópia, parcial ou total, deste documento, nos casos em que é legalmente admitida, deverá conter ou fazer-se acompanhar por este aviso. 
VOLUME $5 \longdiv { 2 0 1 4 }$

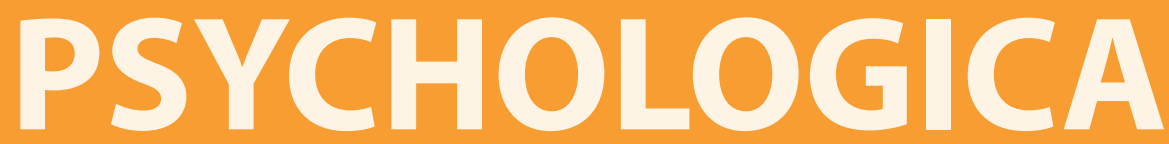

IMPRENSA DA UNIVERSIDADE DE COIMBRA

COIMBRA UNIVERSITY PRESS

FACULDADE DE PSICOLOGIA E DE CIÊNCIAS

DA EDUCAÇÃO DA UNIVERSIDADE DE COIMBRA 


\title{
Paixão pelo trabalho e competências de gestão interpessoal: Validação de escalas para a sua avaliação junto de gestores empresariais
}

\author{
Margarida Martins ${ }^{1}$, Alexandra M. Araújo² e Leandro S. Almeida ${ }^{3}$
}

Passion toward work and political skills: Validation of scales for the assessment of business managers

\begin{abstract}
This study presents the validation of the Passion Toward Work Scale (PTWS; Vallerand \& Houlfort, 2003) and of the Political Skill Inventory (PSI; Ferris, Treadway, Kolodinsky, Hochwarter, Kacmar, Douglas, \& Frink, 2005) for Portuguese business managers. The PTWS consists of 14 items, distributed in two dimensions: obsessive passion and harmonious passion. The PSI consists of 18 items and it includes four dimensions: social astuteness, interpersonal influence, networking ability, and apparent sincerity. There were 131 Portuguese managers participating in the study, from the North, Center and South of Portugal, and from various activity domains, age cohorts, and both genders. Findings suggest that both measures present high levels of precision and that the constructs originally defined by the authors are replicated in the dimensional structure for the Portuguese sample. A statistically significant and positive relation was also found between harmonious passion, assessed with the PTWS, and interpersonal management skills, measured with the PSI.
\end{abstract}

Keywords: business management; passion for work; political skills; assessment

1 Instituto de Educação, Universidade do Minho, PORTUGAL

2 Instituto de Educação, Universidade do Minho, PORTUGAL. A segunda autora foi apoiada pela Fundação para a Ciência e Tecnologia, através da atribuição de uma bolsa de investigação de pós-doutoramento (SFRH/BPD/85856/2012).

3 Instituto de Educação, Universidade do Minho, PORTUGAL. Email: leandro@ie.uminho.pt 


\section{Resumo}

O presente estudo teve como objetivo a validação da Passion Toward Work Scale (PTWS - Escala da Paixão pelo Trabalho; Vallerand \& Houlfort, 2003) e do Political Skill Inventory (PSI - Inventário de Competências de Gestão Interpessoal; Ferris et al., 2005) para gestores empresariais portugueses. A PTWS é uma escala composta por 14 itens, distribuídos por duas dimensões: paixão obsessiva e paixão harmoniosa. O PSI, constituído por 18 itens, integra quatro dimensões: a perspicácia social, a influência interpessoal, a capacidade para estabelecer redes de relacionamento profissional, e a sinceridade genuína. Participaram neste estudo 131 gestores portugueses, do norte ao sul do país, de várias áreas de atividade, faixas etárias e de ambos os sexos. Os resultados encontrados sugerem que ambos os instrumentos apresentam elevados índices de precisão e que os constructos definidos originalmente pelos autores se replicam na sua estrutura dimensional nesta amostra portuguesa. Foi observada, ainda, uma correlação positiva e estatisticamente significativa entre as competências de gestão interpessoal avaliadas pelo PSI e a dimensão paixão harmoniosa do PTWS.

Palavras-chave: gestão empresarial; paixão pelo trabalho; competências de gestão interpessoal; avaliação

\section{INTRODUÇÃO}

O trabalho constitui uma das atividades mais salientes na vida, tendo expressão ao longo de todo o ciclo vital, e sendo através do mesmo que o indivíduo implementa, em boa medida, o seu autoconceito (Super, 1990; Super, Savickas, \& Super, 1996). O trabalho efetuado pelo indivíduo, no desempenho dos vários papéis de vida, traduz a expressão de preferências e interesses de vida, e reflete a forma como o indivíduo se vê a si próprio. Mais ainda, constata-se que, mesmo após a reforma, muitas pessoas continuam envolvidas em atividades relacionadas com o seu antigo emprego, refletindo o peso do trabalho na identidade pessoal e a paixão mais ou menos adaptativa como cada um vivenciou o seu trabalho (Vallerand \& Houlford, 2003).

Vallerand e colaboradores (2003), partindo da definição de paixão como "uma forte inclinação para uma atividade que as pessoas gostam, que acham importante e em que investem tempo e energia" (p. 757), realizaram um estudo com vista à construção de uma escala sobre a paixão no trabalho. Segundo estes autores, uma atividade torna-se numa paixão através de dois processos: em primeiro lugar, pela 
valorização que o sujeito faz da atividade e, em segundo, pela internalização da representação da atividade no self, isto é, na sua identidade, através das relações subjetivas, das comunicações, da linguagem e das experiências sociais (Vallerand et al., 2003; Vallerand \& Houlfort, 2003; Vallerand et al., 2008; Vallerand et al., 2006). Baseando-se nos trabalhos de Aron, Aron e Smollan (1992) e de Csikszentmihalyi, Rathunde e Whalen (1993), Vallerand e colaboradores (2003) propõem que as representações das atividades que as pessoas gostam, e nas quais se envolvem regularmente, irão ser incorporadas na sua identidade, de acordo com a valorização que lhe é atribuída, surgindo deste modo a paixão por essa atividade. Tais paixões tornam-se elementos centrais da identidade do sujeito, servindo a sua definição como pessoa.

O processo de internalização da atividade profissional na identidade do sujeito sugere a existência de dois tipos de paixão, com qualidade psicológica diferente: uma paixão harmoniosa e uma paixão obsessiva (Vallerand et al., 2003; Vallerand et al., 2008; Vallerand et al., 2006). A paixão harmoniosa resulta de uma internalização autónoma da atividade para a identidade da pessoa, isto é, quando o sujeito aceita livremente a atividade como importante para si próprio, sem quaisquer contingências. Este tipo de internalização produz uma força motivacional para exercer a atividade de bom grado e gera um sentimento de vontade e apoio pessoal sobre o exercício da atividade. Com este tipo de paixão, associada a emoções positivas, longos períodos de concentração na tarefa e fluência na produção, a atividade ocupa um espaço significativo, mas não avassalador na identidade da pessoa, e está em harmonia com outros domínios da sua vida. Em contraste, a paixão obsessiva resulta de uma fixação persistente da atividade e sua internalização na identidade do sujeito. Tal internalização resulta da pressão intrapessoal e/ou interpessoal, quer porque certos resultados estão relacionados com a atividade, tais como sentimentos de aceitação social e autoestima, quer porque o entusiasmo derivado da prática da atividade se torna incontrolável e excessivo, estando associada a emoções negativas e provocando conflitos com outras atividades na vida da pessoa (Vallerand et al., 2003; Vallerand \& Houlfort, 2003).

Este duplo significado da paixão proposto por Vallerand e colaboradores (2003) surge nas escalas propostas para a sua avaliação. Uma primeira lista composta por 34 itens incluía autoavaliações que enfatizavam uma perspetiva passiva de paixão, na qual a pessoa se sentia impelida para exercer uma atividade, sem controlo e com a presença de conflitos interiores (obsessive passion - paixão obsessiva), bem como autoavaliações que enfatizavam uma perspetiva ativa da paixão, onde a pessoa sentia controlo sobre a atividade e esta estava em harmonia com outras dimensões da sua vida (harmonious passion - paixão harmoniosa). Neste conjunto de itens foi confirmada a matriz inicial da conceção dualista da paixão proposta 
pelos autores (Vallerand et al., 2003). Foram então selecionados os 14 itens que mais saturavam nos dois fatores identificados, dando origem à Passion Scale (PS, Escala de Paixão; Vallerand et al., 2003), a qual foi posteriormente adaptada para o domínio do trabalho, designando-se Passion Toward Work Scale (PTWS, Escala de Paixão pelo Trabalho; Vallerand \& Houlfort, 2003).

Focando especificamente as competências dos profissionais na área da gestão empresarial, uma outra variável a considerar na investigação serão as competências de gestão interpessoal. Estas competências podem ser entendidas como capacidades de compreender os outros no trabalho e usar esse conhecimento para influenciar os outros de modo a que estes atuem para atingir objetivos pessoais e/ou organizacionais (Ahearn, Ferris, Hochwarter, Douglas, \& Ammeter, 2004). Uma análise cuidadosa da literatura sobre competências organizacionais levou Ferris e colaboradores (2002, 2005) a considerarem quatro aspetos que devem ser incluídos em qualquer medida representativa das competências de gestão interpessoal na prática profissional: a perspicácia social (social astuteness), ou seja, a capacidade de observar os outros com perspicácia e interpretar os seus comportamentos, através de uma profunda sintonia face às diversas situações sociais; a influência interpessoal (interpersonal influence), ou seja, a capacidade de convencer, exercendo poder, os outros em seu redor; a capacidade de estabelecer redes de relacionamento profissional (networking ability), entendida como a capacidade de criar e recorrer a redes de trabalho, que se constituem na base de fortes alianças sociais no trabalho; e a sinceridade genuína (apparent sincerity), isto é, a capacidade para demonstrar competências elevadas de autenticidade, genuinidade, integridade e sinceridade aos outros (Ferris, Anthony, Kolodinsky, Gilmore, \& Harvey, 2002; Ferris et al., 2005; Ferris et al., 2007).

Na construção do Political Skill Inventory (PSI; Inventário de Competências de Gestão Interpessoal; Ferris et al., 2005) foi gerada uma lista com 40 itens representativos dos quatro domínios de competências de gestão interpessoal no quadro da atividade profissional: perspicácia social (e.g., "Sou particularmente bom a captar as motivações e as segundas intenções dos outros”), influência interpessoal (e.g., "Para mim é fácil estabelecer um bom relacionamento com a maioria das pessoas"), capacidade de estabelecer redes de relacionamento profissional (e.g., "Sou bom a usar a minha rede de contactos para fazer as coisas acontecerem no trabalho") e sinceridade genuína (e.g., "Quando comunico com os outros, procuro ser verdadeiro/a no que digo e faço”). A versão final (Ferris et al., 2005), constituída por 18 itens, indica a valorização básica que o indivíduo faz sobre as suas competências de gestão interpessoal, tendo-se em vários estudos validado as quatro dimensões representativas de tais competências (Bing, Davison, Minor, Novicevic, \& Frink, 2011; Blickle et al., 2012; Blickle et al., 2011; Ferris et al., 2005; Ferris et al., 2007; Treadway et al., 2013). 
O presente estudo teve como objetivo a validação dos dois instrumentos descritos, a Passion Toward Work Scale (PTWS; Escala da Paixão pelo Trabalho; Vallerand \& Houlfort, 2003) e o Political Skill Inventory (PSI; Inventário de Competências de Gestão Interpessoal; Ferris et al., 2005) para gestores empresariais portugueses. Previamente à recolha de dados para o presente estudo, assegurou-se junto dos autores a autorização para a realização deste estudo, assegurando também que as mesmas escalas não haviam ainda sido validadas em Portugal até à data.

\section{MÉTODO}

\section{Participantes}

Participaram neste estudo 131 gestores de ambos os sexos, sendo $47 \%$ homens e $53 \%$ mulheres, com idades compreendidas entre os 24 e os 72 anos $(M=43.4 ; D P$ =9.64), e de diversos domínios de atividade económica, designadamente indústria, comércio, militar, saúde e educação. Nesta amostra, 75\% dos participantes têm como habilitações académicas o ensino superior e 65\% são casados. Estes participantes residem em várias zonas geográficas do país, sendo $42 \%$ do sul, 28\% do norte, 18\% do centro. Refira-se ainda que $13 \%$ dos participantes, apesar de terem nacionalidade portuguesa, nasceram noutros países (e.g., França, Angola, Brasil). A idade em que os participantes iniciaram a sua atividade como gestores oscila entre os 17 e os 55 anos $(M=29.17 ; D P=6.36)$. Por outro lado, $42 \%$ são profissionais que trabalham em grandes empresas (mais de 250 colaboradores), 22\% em médias empresas (50 a 250 colaboradores), $9 \%$ em pequenas empresas (10 a 49 colaboradores), e $27 \%$ em microempresas (menos de 10 colaboradores).

\section{Instrumentos}

A Passion Toward Work Scale (Vallerand \& Houlfort, 2003) resulta da adaptação da Passion Scale (PS; Vallerand et al., 2003) para o trabalho, avaliando duas formas de paixão: uma paixão harmoniosa, associada a emoções positivas, e uma paixão obsessiva, associada a emoções negativas e a conflitos com outras dimensões da vida do sujeito. Do total de 14 itens, sete itens pertencem ao fator Paixão Obsessiva e sete itens ao fator Paixão Harmoniosa, sendo as respostas dadas numa escala tipo 
Likert de 7 pontos variando entre 1 ("totalmente em desacordo") e 7 ("totalmente de acordo"). Os valores de precisão (alfa de Cronbach) encontrados para a Passion Toward Work Scale (PTWS) nos estudos de validação inicial junto da população norte-americana situaram-se entre .70 e .85 (Vallerand \& Houlford, 2003).

O Political Skill Inventory (PSI; Ferris et al., 2005) é um instrumento multidimensional para a avaliação das competências de gestão interpessoal, entendida como capacidades de compreender e influenciar os outros, para a concretização de objetivos pessoais e/ou organizacionais. A versão final, constituída por 18 itens, avalia quatro fatores: Perspicácia Social, Influência Interpessoal, Capacidade de Estabelecer Redes de Relacionamento Profissional e Sinceridade Genuína. As respostas são fornecidas numa escala tipo Likert de 7 pontos variando entre 1 ("totalmente em desacordo") e 7 ("totalmente de acordo"). Ferris e colaboradores (2005) obtiveram para cada fator, Influência Interpessoal, Perspicácia Social, Sinceridade Genuína e Capacidade de Estabelecer Redes de Relacionamento Profissional, coeficientes de consistência interna (alfa de Cronbach) respetivamente de .78, .79, .81 e .87.

\section{Procedimentos}

Dado que ambos os instrumentos, PTWS e PSI, ainda não se encontravam traduzidos em Portugal à data da recolha de dados, procedemos à sua tradução e retrotradução recorrendo a psicólogos com elevada fluência na língua inglesa. Com o objetivo de aumentar a validade da escala, recorremos ao método da reflexão falada junto de cinco gestores empresariais. Estes gestores, dois homens e três mulheres, com idades compreendidas entre 30 e 50 anos, trabalhavam em microempresas e tinham uma experiência profissional de mais de dez anos de gestão. Nesta reflexão falada, procurámos recolher informação acerca da acessibilidade do vocabulário utilizado na escala e garantir a compreensão unívoca das suas instruções e dos seus itens. Procurámos, também, obter uma estimativa do tempo requerido para o preenchimento do instrumento. Durante todo o processo da reflexão falada procedeu-se ao registo dos comentários dos participantes, designadamente dúvidas quanto ao conteúdo dos itens, das instruções para o preenchimento da escala e das questões sobre os dados de caracterização. As alterações introduzidas em resultado da reflexão falada incidiram sobre a tradução e clarificação dos itens.

Juntamente com as escalas PTWS e PSI, foi administrada uma breve ficha de caracterização sociodemográfica. A recolha de dados decorreu entre abril e julho de 2013, numa amostra de conveniência constituída pelo efeito "bola de neve", tendo-se optado quer pela resposta online, quer pela resposta em suporte de papel. A análise das características psicométricas de ambas as escalas foi efetuada com recurso ao 
programa estatístico IBM SPSS Statistics, versão 22.0 para Windows. A precisão foi estimada através da consistência interna dos itens (alfa de Cronbach) e a análise da dimensionalidade das duas escalas foi efetuada através da análise fatorial exploratória pelo método das componentes principais, com rotação promax dos componentes isolados, em virtude de teoricamente os fatores isolados estarem correlacionados, tendo-se recorrido à regra de retenção de fatores com valor-próprio igual ou superior à unidade (princípio de Kaiser) e fixando em .40 o valor mínimo de saturação dos itens. Para as intercorrelações entre as dimensões das duas escalas, verificada a distribuição normal das pontuações, recorreu-se ao coeficiente de correlação produto $\mathrm{x}$ momento de Pearson, assumindo como limiar crítico da significância $p<.05$.

\section{Resultados}

A tabela 1 apresenta os resultados da análise fatorial dos itens da Escala da Paixão pelo Trabalho (PTWS). Para a análise da adequabilidade da amostra e determinação da fatorização dos itens aplicámos o teste Kaiser-Meyer-Olkin, tendo-se obtido um coeficiente $K M O=.84$, bem como o teste de esfericidade de Bartlett, com um resultado estatisticamente significativo $\left(\chi^{2}=1053.631 ; g l=91 ; p<0.001\right)$. Ambos os índices são favoráveis à fatorização dos itens.

Foram identificados três fatores com valor próprio superior à unidade, sendo que o primeiro fator explica $39.8 \%$ da variância, o segundo fator explica $20.3 \%$, e o terceiro apenas 7.8\%. Este último fator aparece basicamente associado ao item "O meu estado de humor depende da minha capacidade de fazer o meu trabalho", com uma saturação de .92. Existem dois outros itens que estão associados a este fator e simultaneamente ao Fator 1. Dada a teoria que sustenta a avaliação da paixão pelo trabalho e dado o sentido residual deste terceiro fator, optou-se por conduzir uma nova análise fatorial, especificando dois fatores.

Tabela 1

Resultados da Análise Fatorial dos Itens da Escala da Paixão pelo Trabalho, PTWS (N=131)

\begin{tabular}{|c|c|c|c|c|}
\hline Itens & F1 & F2 & $h^{2}$ & ritc \\
\hline $\begin{array}{l}1 \text { - O meu trabalho dá-me oportunidade de viver uma diversi- } \\
\text { dade de experiências. }\end{array}$ & & .69 & .47 & .60 \\
\hline $\begin{array}{l}2 \text { - As coisas novas que descubro no meu trabalho permitem- } \\
\text {-me apreciá-lo ainda mais. }\end{array}$ & & .83 & .65 & .70 \\
\hline $\begin{array}{l}3 \text { - A minha área de trabalho está de acordo com as qualidades } \\
\text { que aprecio em mim. }\end{array}$ & & .82 & .63 & .68 \\
\hline $\begin{array}{l}4 \text { - O meu trabalho está em harmonia com as outras atividades } \\
\text { da minha vida. }\end{array}$ & & .65 & .39 & .49 \\
\hline
\end{tabular}


5 - O meu trabalho é uma paixão que ainda sou capaz de controlar.

6 - O meu trabalho permite-me viver experiências memoráveis.

7 - Estou completamente envolvido/a no meu trabalho.

$\begin{array}{lll}.65 & .59 & .64\end{array}$

8 - Não consigo viver sem o meu trabalho.

9 - O desejo é tão forte que não consigo evitar de trabalhar.

10 - Tenho dificuldade em imaginar a minha vida sem o meu trabalho.

11 - Estou emocionalmente dependente do meu trabalho.

12 - Tenho dificuldades em controlar o meu impulso para fazer o meu trabalho.

13 - Tenho um sentimento quase obsessivo pelo meu trabalho.

14 - O meu estado de humor depende da minha capacidade de fazer o meu trabalho.

Através da leitura da tabela 1 , podemos observar que $60.1 \%$ da variância dos itens é explicada pelos dois fatores identificados, sendo esta percentagem elevada, tal como verificado nos estudos norte-americanos. Por sua vez, os valores próprios são de 5.57 (Fator 1) e 2.84 (Fator 2). Os itens apresentam comunalidades acima de .40, exceto no item 14 ("O meu estado de humor depende da minha capacidade de fazer o meu trabalho"), cuja comunalidade se situa em .25 , sugerindo tratar-se de um item mais específico ou pouco relacionado com os dois fatores identificados.

Tal como na versão norte-americana (Vallerand \& Houlford, 2003), os itens distribuem-se pelos dois fatores, Paixão Harmoniosa e Paixão Obsessiva, seguindo a mesma organização dimensional. Relativamente à precisão de cada fator encontrado, os valores são bastante superiores a .60, índice mínimo requerido em estudos exploratórios de construção de instrumentos (Nunnally, 1978). Analisando a precisão das subescalas, o Fator 2, constituído por sete itens (itens 1 a 7), apresenta um alfa de Cronbach de .86, sendo que as correlações dos itens com o total da escala oscilam entre .49 (no item 4 ) e .70 (no item 2). O Fator 1, constituído por sete itens (itens 8 a 14), apresenta um alfa de Cronbach de .90, observando-se que as correlações dos itens com o total da escala oscilam entre .37 (no item 14) e .80 (no item 11). Neste caso concreto, se o item 14 for eliminado o valor do alfa sobe para .92. No entanto, dados os valores positivos obtidos, e na lógica de não alterar desnecessariamente a escala, optámos por manter este item na versão portuguesa.

A tabela 2 apresenta os resultados da análise fatorial dos itens do Inventário de Competências de Gestão Interpessoal (PSI). Para a análise da adequabilidade da 
amostra e determinação da fatorização dos itens aplicámos o teste Kaiser-Meyer-Olkin, obtendo-se um índice de $K M O=.86$, e o teste de esfericidade de Bartlett com um resultado estatisticamente significativo $\left(\chi^{2}=1150.881 ; g l=153 ; p<0.001\right)$. Ambos os índices são favoráveis à fatorização dos itens. A análise fatorial exploratória identifica quatro fatores com valor próprio igual ou superior à unidade, explicando no seu conjunto $65 \%$ da variância. A tabela 2 apresenta, ainda, os índices alfa de Cronbach para cada fator ou dimensão identificada e, também, os coeficientes de correlação corrigida dos itens com o total da respetiva dimensão (ritc).

Tabela 2

Resultados da Análise Fatorial dos Itens do Inventário de Competências de Gestão Interpessoal, PSI $(N=131)$

\begin{tabular}{|c|c|c|c|c|c|c|}
\hline Itens & F1 & F2 & F3 & F4 & $h^{2}$ & ritc \\
\hline $\begin{array}{l}1 \text { - Despendo muito tempo e esforço a criar } \\
\text { redes de trabalho com os outros colegas. }\end{array}$ & .88 & & & & .66 & .62 \\
\hline $\begin{array}{l}2 \text { - No trabalho, conheço muitas pessoas im- } \\
\text { portantes e estou bem relacionado/a. }\end{array}$ & .75 & & & & .59 & .65 \\
\hline $\begin{array}{l}3 \text { - Sou bom a usar a minha rede de contactos } \\
\text { para fazer as coisas acontecerem no trabalho. }\end{array}$ & .85 & & & & .75 & .75 \\
\hline $\begin{array}{l}4 \text { - Desenvolvi uma grande rede de contactos } \\
\text { no trabalho, a quem eu posso pedir apoio, } \\
\text { quando realmente preciso fazer as coisas. }\end{array}$ & 84 & & & & .64 & .69 \\
\hline $\begin{array}{l}5 \text { - Passo muito tempo no trabalho a desen- } \\
\text { volver contactos com outros. }\end{array}$ & .67 & & & & .54 & .64 \\
\hline $\begin{array}{l}6 \text { - Sou bom a construir relacionamentos com } \\
\text { pessoas influentes no trabalho. }\end{array}$ & .72 & & & & .75 & .76 \\
\hline $\begin{array}{l}10 \text { - Instintivamente sei dizer ou fazer as coisas } \\
\text { certas para influenciar os outros. }\end{array}$ & & .39 & & & .44 & .53 \\
\hline $\begin{array}{l}11 \text { - Tenho uma boa intuição ou habilidade } \\
\text { sobre como me apresentar aos outros. }\end{array}$ & & .51 & & & .70 & .70 \\
\hline $\begin{array}{l}15 \text { - Para mim é fácil estabelecer um bom rela- } \\
\text { cionamento com a maioria das pessoas. }\end{array}$ & & .90 & & & .69 & .62 \\
\hline $\begin{array}{l}16 \text { - Sou capaz de fazer a maioria das pessoas } \\
\text { sentir-se confortável e à vontade em meu redor. }\end{array}$ & & .88 & & & .76 & .77 \\
\hline $\begin{array}{l}17 \text { - Sou capaz de comunicar com facilidade e } \\
\text { eficazmente com os outros. }\end{array}$ & & .76 & & & .65 & .68 \\
\hline $\begin{array}{l}18 \text { - Sou bom a conseguir que as pessoas gos- } \\
\text { tem de mim. }\end{array}$ & & .68 & & & .55 & .64 \\
\hline $\begin{array}{l}7 \text { - Para mim é importante que as pessoas } \\
\text { acreditem que eu sou sincero/a naquilo que } \\
\text { digo e faço. }\end{array}$ & & & .71 & & .61 & .45 \\
\hline $\begin{array}{l}8 \text { - Quando comunico com os outros, procuro } \\
\text { ser verdadeiro/a no que digo e faço. }\end{array}$ & & & .78 & & .68 & .62 \\
\hline $\begin{array}{l}9 \text { - Procuro mostrar um interesse genuíno } \\
\text { pelas outras pessoas. }\end{array}$ & & & .70 & & .68 & .46 \\
\hline
\end{tabular}


12 - Sou particularmente bom a captar as motivações e as segundas intenções dos outros.

$.67 \quad .72 \quad .56$

13 - Presto atenção às expressões faciais das pessoas.

14 - Compreendo as pessoas muito bem.

\% de variância explicada

$\begin{array}{llll}37.7 & 10.7 & 10.1 & 6.6\end{array}$

Valor próprio dos fatores

$\begin{array}{llll}6.79 & 1.92 & 1.81 & 1.19\end{array}$

Alfa de Cronbach

$\begin{array}{llll}.88 & .86 & .67 & .69\end{array}$

Observando os resultados obtidos na tabela 2, podemos verificar que os quatro fatores explicam $65.1 \%$ da variância dos itens, sendo este valor próximo do obtido nos estudos norte-americanos (Ferris et al., 2005). Por sua vez, os valores próprios situam-se entre 6.79 (Fator 1) e 1.19 (Fator 4), com uma percentagem de variância explicada entre $37.7 \%$ e $6.6 \%$ respetivamente. O item 10 ("Instintivamente sei dizer ou fazer as coisas certas para influenciar os outros") apresenta-se no limiar de saturação mínimo exigido (.39), integrando-se no Fator 2. As comunalidades variam entre .44 (item 10) e .76 (item 16). Seguindo a organização dimensional da escala na sua versão original, o Fator 1 , Capacidade de Estabelecer Redes de Relacionamento Profissional, e o Fator 3, Sinceridade Genuína, são constituídos pelos mesmos itens da versão norte-americana. Associado ao Fator 2, Influência Interpessoal, surgem mais dois itens (item 10, "Instintivamente sei dizer ou fazer as coisas certas para influenciar os outros"; e item 11, "Tenho uma boa intuição ou habilidade sobre como me apresentar aos outros") que na amostra norte-americana estavam associados ao fator Perspicácia Social.

Relativamente à consistência interna dos itens em cada fator, os coeficientes obtidos foram satisfatórios. O Fator 1, Capacidade de Estabelecer Redes de Relacionamento Profissional, inclui seis itens (itens 1 a 6), apresenta um alfa de Cronbach de .88, e as correlações dos itens com o total desse fator oscilam entre .62 (item 1) e .76 (item 6). O Fator 2, Influência Interpessoal, com seis itens (itens 10, 11 e de 15 a 18) apresenta um alfa de Cronbach de .86 e as correlações dos itens com o total desse fator situaram-se entre .53 (item 10) e .77 (item 16). O Fator 3, Sinceridade Genuína), com três itens (itens $7,8,9$ ), apresenta um alfa de Cronbach de .67, e as correlações dos itens com o total desse fator oscilaram entre .45 (item 7 ) e .62 (item 8). O Fator 4 , Perspicácia social, inclui três itens (itens 12, 13, 14), apresenta um alfa de Cronbach de .69 , e as correlações dos itens com o total desse fator situaram-se entre .45 (item 13) e .56 (item 12).

Atendendo a que os participantes responderam a ambas as escalas descritas, e no sentido de obter informação complementar para a sua validação, procurámos conhecer a associação entre as pontuações nas duas escalas. A tabela 3 apresenta 
os coeficientes de correlação obtidos. Observa-se que os índices de correlação das pontuações dos participantes nas duas escalas foram claramente diferenciados de acordo com o tipo de paixão que se considera na análise. As correlações são moderadas, mas sempre significativas do ponto de vista estatístico, quando cruzamos a dimensão da Paixão Harmoniosa com as dimensões Capacidade para Estabelecer Redes de Relacionamento Profissional (.34), Sinceridade Genuína (.25), Perspicácia Social (.36), e Influência Interpessoal (.39).

Tabela 3

Coeficientes de Correlação Entre as Dimensões da PTWS e PSI $(N=131)$

\begin{tabular}{|c|c|c|c|c|c|c|c|}
\hline Dimensão & $M$ & $D P$ & 2. & 3. & 4. & 5. & 6. \\
\hline 1. Paixão obsessiva & 26.05 & 9.81 & $.32^{\star * *}$ & .13 & .14 & -.01 & .05 \\
\hline 2. Paixão harmoniosa & 38.95 & 6.28 & & $.34^{\star * *}$ & $.39^{* * *}$ & $.25^{\star *}$ & $.36^{\star * *}$ \\
\hline 3. Capacidade estabelecer redes & 29.18 & 6.10 & & & $.55^{\star * *}$ & $.27^{\star *}$ & $.45^{\star * *}$ \\
\hline 4. Influência interpessoal & 32.85 & 4.42 & & & & $.26^{\star *}$ & $.57^{\star \star *}$ \\
\hline 5. Sinceridade genuína & 19.47 & 1.73 & & & & & $.33^{* * *}$ \\
\hline 6. Perspicácia social & 16.26 & 2.28 & & & & & 1.00 \\
\hline
\end{tabular}

\section{Discussão}

O objetivo deste estudo foi o de adaptar e validar a Passion Toward Work Scale (PTWS; Vallerand \& Houlford, 2003) e o Political Skill Inventory (PSI; Ferris et al., 2005) para gestores portugueses, aprofundando-se ainda a relação entre a paixão pelo trabalho (harmoniosa e obsessiva) e as competências de gestão interpessoal (capacidade de estabelecer redes de relacionamento interpessoal, influência interpessoal, sinceridade genuína e perspicácia social). Ambos os instrumentos avaliam variáveis que influenciam a satisfação, bem-estar e eficácia profissional, e que são particularmente relevantes no caso de gestores. A paixão pelo trabalho, e particularmente a paixão harmoniosa, contrariamente à paixão obsessiva, tem estado associada a níveis mais elevados de persistência, envolvimento na tarefa, flexibilidade, vitalidade, realização, bem-estar e ajustamento psicológico, que constituem importantes recursos pessoais para que os gestores possam fazer face às exigências do seu trabalho, na liderança de equipas e na resolução de problemas, no presente contexto empresarial e organizacional altamente competitivo (Forest, Mageau, Sarrazin, \& Morin, 2011; Trépanier, Fernet, Austin, Forest, \& Vallerand, 2014; Vallerand \& Houlfort, 2003). Por seu lado, indivíduos com elevadas competências de gestão interpessoal obtêm melhores resultados no seu trabalho, em larga medida devido ao facto de possuírem 
uma boa rede de relações profissionais, na qual têm uma boa reputação, e através da qual acedem a informação fulcral para a tomada de decisão (Ferris et al., 2005; Liu et al., 2007). Tais indivíduos parecem apresentar um sentido de controlo sobre o seu ambiente de trabalho, neutralizando o efeito negativo dos stressores ou crises (Ferris et al., 2005; Meurs, Gallagher, \& Perrewé, 2010). Dada a relevância destes dois constructos para a eficácia no relacionamento interpessoal, persistência, bem- estar pessoal, flexibilidade e adaptação a situações de stress, é importante dispor de instrumentos de medida fiáveis para a sua avaliação, nomeadamente em contexto organizacional. Tais instrumentos poderão auxiliar a melhor compreender e avaliar a eficácia da gestão e liderança de equipas, potencialmente servindo como instrumentos de diagnóstico e de avaliação da intervenção procurando uma melhor correspondência entre as características disposicionais dos gestores e o tipo de trabalho que lhes é requerido, e maximizando os seus níveis de realização e os da sua organização.

Os coeficientes de consistência interna dos itens e sua dimensionalidade, quer para a PTWS quer para o PSI, são adequados e atestam as qualidades psicométricas dos dois instrumentos de avaliação psicológica em Portugal, replicando-se em larga medida os valores obtidos com a versão original na população norte-americana. Este estudo com gestores portugueses mostra que a paixão pelo trabalho se diferencia em duas dimensões, tal como proposto no modelo dualista da paixão (Vallerand et al., 2003): Paixão Obsessiva e Paixão Harmoniosa. A representação fatorial obtida neste estudo com a Escala da Paixão pelo Trabalho (PTWS), tal como na versão original (Vallerand \& Houlfort, 2003), mostra-se assim adequada para a avaliação deste constructo. Os índices de consistência interna para a Paixão Obsessiva (a Cronbach $=.90$ ) e Paixão Harmoniosa ( $\alpha$ Cronbach $=.86$ ) são elevados, atingindo inclusivamente valores superiores aos encontrados em estudos prévios junto da população norte-americana (Forest et al., 2011; Vallerand \& Houlfort, 2003).

No caso desta amostra de gestores, a Paixão Harmoniosa obtém, em média, resultados superiores à Paixão Obsessiva, o que constitui um resultado positivo em termos de bem-estar geral e no trabalho. As duas manifestações da paixão pelo trabalho mostram-se moderadamente correlacionadas $(r=.32 ; p<.001)$, à semelhança do observado em estudos prévios, uma vez que ambas indiciam a valorização da atividade em si e o tempo despendido na mesma, neste caso o trabalho (Vallerand et al., 2006; Vallerand \& Houlfort, 2003).

Por seu turno, no Inventário de Competências de Gestão Interpessoal (PSI) surgem, tal como nos estudos norte-americanos, quatro fatores: Capacidade de Estabelecer Redes de Relacionamento Profissional, Perspicácia Social, Sinceridade Genuína, e Influência Interpessoal. A organização dos itens em torno destas quatro dimensões reproduz o estudo original de validação do PSI (Ferris et al., 2005). Contudo, nesta amostra de gestores portugueses, o item 10 ("Instintivamente sei dizer ou fazer as 
coisas certas para influenciar os outros") e o item 11 ("Tenho uma boa intuição ou habilidade sobre como me apresentar aos outros") surgem associados ao Fator 2 (Influência Interpessoal). No caso do item 10, esta situação parece-nos bastante óbvia dada a formulação do item, enquanto no caso do item 11 depreende-se que, subjacente à apresentação pessoal, os gestores portugueses terão uma intenção de influenciar os outros. Os índices de consistência interna são aceitáveis no caso das dimensões Capacidade de Estabelecer Redes de Relacionamento Profissional (F1; $a$ Cronbach $=.88)$ e Influência Interpessoal $(\mathrm{F} 2$; a Cronbach $=.86)$, aproximando-se dos valores encontrados por Ferris e colaboradores (2005). Tais índices são, no entanto, inferiores no caso das dimensões Sinceridade Genuína (F3; a Cronbach $=.67)$ e Perspicácia Social $(F 4 ;$ a Cronbach $=.69)$, o que poderá estar associado ao menor número de itens que integra cada fator, neste caso apenas três itens em cada dimensão. Finalmente, é importante notar que as correlações encontradas entre as dimensões avaliadas pelo PSI são moderadas, uma vez mais, tal como no estudo de Ferris e colaboradores (2005).

O estudo da validade de critério das dimensões avaliadas pela PTWS e pelo PSI mostrou que, neste grupo de gestores portugueses, as dimensões da paixão pelo trabalho apresentam uma relação claramente diferenciada com as dimensões avaliadas das competências de gestão interpessoal: enquanto a Paixão Harmoniosa correlaciona moderada e positivamente com as mesmas, tal não acontece no caso da Paixão Obsessiva. Este resultado parece estar em consonância com o tipo de paixão harmoniosa e obsessiva que se avalia na PTWS. As competências pessoais de relacionamento interpessoal avaliadas no PSI pressupõem um bom autocontrolo do gestor, até como ponto de partida para um adequado controlo e influência dos seus colaboradores. Assim, as relações encontradas neste estudo reforçam os resultados da investigação que reporta uma associação significativa entre as competências de gestão interpessoal e a capacidade de gestão emocional e do stress, tal como é característico na paixão harmoniosa (Carbonneau, Vallerand, Fernet, \& Guay, 2008; Ferris et al., 2005; Meurs et al., 2010). Os gestores que fazem uma gestão mais eficaz das suas emoções e do valor atribuído ao seu trabalho - paixão harmoniosa - parecem ser também aqueles que têm uma maior capacidade de monitorizar o seu comportamento e adaptá-lo às situações sociais, que estão mais atentos às situações sociais, que fazem uma melhor interpretação do seu próprio comportamento e do comportamento dos outros, e que apresentam um maior conhecimento de si mesmos. Mais especificamente, a este respeito, poder-se-á inferir que os gestores que apresentam simultaneamente níveis mais elevados de paixão harmoniosa pelo trabalho e de competências de gestão interpessoal, dada a sua flexibilidade, autoconhecimento e capacidades de autocontrolo e de influência interpessoal, terão melhores competências de gestão do stress (Ferris et al., 2005). 
Em suma, terminamos com o reconhecimento da importância, a par de competências técnicas, de competências pessoais e interpessoais para o exercício das funções de liderança e gestão, em contexto grupal e organizacional. A qualificação em termos do controlo da paixão pelo trabalho e da competência na influência do comportamento dos outros é relevante para funções de liderança, como é o caso dos gestores. Mais ainda, umas e outras estão associadas a níveis mais elevados quer de ajustamento psicológico, quer de desempenho profissional (Carbonneau et al., 2008; Cid \& Louro, 2010; Ferris et al., 2005; Houlfort \& Vallerand, 2006; Liu et al., 2007; Mageau et al., 2009; Meurs et al., 2010; Rousseau \& Vallerand, 2003, 2008; Vallerand \& Houlfort, 2003), e por isso constituem variáveis centrais à produtividade em contexto organizacional.

Finalmente, os resultados deste estudo poderão estar influenciados pela baixa dimensão e consequentemente representatividade da amostra considerada, pelo que se recomenda o seu alargamento a amostras mais robustas. Estudos futuros poderão também aprofundar a relação entre a paixão pelo trabalho e as competências de gestão interpessoal e a natureza das características de liderança dos gestores, e como estes inspiram confiança, exercem influência e modelam o comportamento nos seus pares e subordinados. Por outro lado, seria também importante avaliar diferenças nos graus de paixão pelo trabalho e na demonstração de competências de gestão interpessoal em função das áreas profissionais dos gestores, e em função da atividade de gestão ser acumulada ou não com a atividade empresarial (neste caso de empresa própria) do gestor. Também se recomenda a recolha de informação de outras fontes (superiores, colegas e subordinados), dado que, tal como mostra o estudo de Meurs e colaboradores (2010), as auto- e heteroavaliações destas competências produzem resultados diferentes na predição dos níveis de desempenho.

\section{AGRADECIMENTOS / AKNOWLEDGEMENTS}

Os autores agradecem as sugestões e os contributos dos Revisores da primeira versão do artigo enviado, as quais permitiram melhorar significativamente este trabalho.

\section{REFERÊNCIAS}

Ahearn, K., Ferris, G., Hochwarter, W., Douglas, C., \& Ammeter, A. (2004). Leader political skill and team performance. Journal of Management, 30, 309-327. 
Aron, A., Aron, E. N., \& Smollan, D. (1992). Inclusion of other in the self scale and the structure of interpersonal closeness. Journal of Personality and Social Psychology, 63, 596-612.

Bing, M. N., Davison, H. K., Minor, I., Novicevic, M. M., \& Frink, D. D. (2011). The prediction of task and contextual performance by political skill: A meta-analysis and moderator test. Journal of Vocational Behavior, 79, 563-577.

Blickle, G., John, J., Ferris, G. R., Momm, T., Liu, Y., Haag, R., ... Oerder, K. (2012). Fit of political skill to the work context: A two-study investigation. Applied Psychology, 61, 295-322.

Blickle, G., Kramer, J., Schneider, P. B., Meurs, J. A., Ferris, G. R., Mierke, J., .. Momm, T. D. (2011). Role of political skill in job performance prediction beyond general mental ability and personality in cross-sectional and predictive studies. Journal of Applied Social Psychology, 41, 239-265.

Carbonneau, N., Vallerand, R. J., Fernet, C., \& Guay, F. (2008). The role of passion for teaching in intrapersonal and interpersonal consequences. Journal of Educational Psychology, 4, 977-987.

Cid, L., \& Louro, H. (2010). Praticar natação é uma paixão ou um sacrifício? Estudo da relação entre o tipo de paixão que o atleta sente pela modalidade e a sua orientação motivacional. Revista Iberoamericana de Psicología del Ejercicio y el Deporte, 5, 99-113.

Csikszentmihalyi, M., Rathunde, K., \& Whalen, S. (1993). Talented teenagers. The roots of success and failure. New York: Cambridge University Press.

Ferris, G. R., Anthony, W. P., Kolodinsky, R. W., Gilmore, D. C., \& Harvey, M. G. (2002). Development of political skill. In C. Wankel \& R. de Fillippi (Eds.), Research in management education and development, Volume 1: Rethinking management education for the 21st century (pp. 3-25). Greenwich, CT: Information Age Publishing.

Ferris, G. R., Treadway, D. C., Kolodinsky, R. W., Hochwarter, W. A., Kacmar, C. J., Douglas, C., \& Frink, D. D. (2005). Development and validation of the political skill inventory. Journal of Management, 31, 126-152.

Ferris G. R., Treadway D. C., Perrewé, P. L., Brouer, R. L., Douglas, C., \& Lux, S. (2007). Political skill in organizations. Journal of Management, 33, 290-320

Forest, J., Mageau, G. A., Sarrazin, C., \& Morin, E. M. (2011). "Work is my passion": The different affective, behavioural, and cognitive consequences of harmonious and obsessive passion toward work. Canadian Journal of Administrative Sciences, 28, 27-40.

Houlfort, N., \& Vallerand, R. J. (2006). La passion envers le travail: Les deux côtés de la médaille. Revue Multidisciplinaire sur l'Emploi, le Syndicalisme et le Travail, 2, 4-17.

Liu, Y., Ferris, G. R., Zinko, R., Perrewé, P. L., Weitz, B., \& Xu, J. (2007). Dispositional antecedents and outcomes of political skill in organizations: A four-study investigation with convergence. Journal of Vocational Behavior, 71, 146-165.

Mageau, G. A., Vallerand, R. J., Charest, J., Salvy, S. J., Lacaille, N., Bouffard, T., \& Koestner, R. (2009). On the development of harmonious and obsessive passion: The role of autonomy support, activity specialization, and identification with the activity. Journal of Personality, 77, 601-647.

Meurs, J. A., Gallagher, V. C., \& Perrewé, P. L. (2010). The role of political skill in the stressor-outcome relationship: Differential predictions for self- and other-reports of political skill. Journal of Vocational Behavior, 76, 520-533.

Nunnally, J. C. (1978). Psychometric theory (2 $2^{\text {nd }}$ ed.). New York: McGraw-Hill.

Rousseau, F. L., \& Vallerand, R. J. (2003). Le role de la passion dans le bien-être subjectif des aínês. Revue Québécoise de Psychologie, 24, 197-211.

Rousseau, F. L., \& Vallerand, R. J. (2008). An examination of the relationship between passion and subjective well-being in older adults. International Journal of Aging and Human Development, 66, 195-211. 
Super (1990). A life-span, life-space approach to career development. In D. Brown \& L. Brooks (Eds.), Career choice and development: applying contemporary theories to practice ( $2^{\text {nd }}$ ed., pp. 197-261). San Francisco, CA: Jossey Bass.

Super, D. E., Savickas, M. L., \& Super, C. M. (1996). The life-span, life-space approach to careers. In D. Brown \& L. Brooks (Eds.), Career choice and development (pp. 121-178). San Francisco, CA: Jossey-Bass.

Treadway, D., Breland, J., Williams, L., Cho, J., Yang, J., \& Ferris, G. (2013). Social influence and interpersonal power in organizations: Roles of performance and political skill in two studies. Journal of Management, 39, 1529-1553.

Trépanier, S. G., Fernet, C., Austin, S., Forest, J., \& Vallerand, R. J. (2014). Linking job demands and resources to burnout and work engagement: Does passion underlie these differential relationships? Motivation and Emotion, 38, 353-366.

Vallerand, R. J., \& Houlfort, N. (2003). Passion at work: Toward a new conceptualization. In D. Skarlicki, S. Gilliland, \& D. Steiner (Eds.), Social issues in management, Vol. 3. (pp. 175-204). Greenwich, CT: Information Age Publishing.

Vallerand, R. J., Blanchard, C. M., Mageau, G. A., Koestner, R., Ratelle, C., Leonard, M., Gagné, M., \& Marsolais, J. (2003). Les passions de l'âme: On obsessive and harmonious passion. Journal of Personality and Social Psychology, 85, 756-767.

Vallerand, R. J., Mageau, G. A., Elliot, A. J., Dumais, A., Demers, M., \& Rousseau, F. (2008). Passion and performance attainment in sport. Psychology of Sport and Exercise, 9, 373-392.

Vallerand, R. J., Rousseau, F. L., Grouzet, F. M. E., Dumais, A., Grenier, S., \& Blanchard, C. B. (2006). Passion in sport: A look at determinants and affective experiences. Journal of Sport \& Exercise Psychology, 28, 454-478. 